\title{
Identification of Plastoglobules as a Site of Carotenoid Cleavage
}

\author{
Sarah Rottet ${ }^{1}$, Julie Devillers ${ }^{1}$, Gaétan Glauser ${ }^{2}$, Véronique Douet ${ }^{1}$, Céline Besagni ${ }^{1}$ and \\ Felix Kessler ${ }^{1 *}$ \\ ${ }^{1}$ Laboratory of Plant Physiology, Institute of Biology, University of Neuchâtel, Neuchâtel, Switzerland, ${ }^{2}$ Neuchâtel Platform of \\ Analytical Chemistry, University of Neuchâtel, Neuchâtel, Switzerland
}

\section{OPEN ACCESS}

Edited by:

Gustavo Bonaventure, BASF Plant Science, Belgium

Reviewed by:

Shan Lu,

Nanjing University, China

Ján A. Miernyk,

University of Missouri, USA

${ }^{*}$ Correspondence:

Felix Kessler

felix.kessler@unine.ch

Specialty section:

This article was submitted to

Plant Metabolism

and Chemodiversity,

a section of the journal

Frontiers in Plant Science

Received: 09 September 2016 Accepted: 24 November 2016 Published: 08 December 2016

Citation:

Rottet S, Devillers J, Glauser G,

Douet V, Besagni $C$ and Kessler $F$ (2016) Identification of Plastoglobules

as a Site of Carotenoid Cleavage.

Front. Plant Sci. 7:1855.

doi: 10.3389/fpls.2016.01855
Carotenoids play an essential role in light harvesting and protection from excess light. During chloroplast senescence carotenoids are released from their binding proteins and are eventually metabolized. Carotenoid cleavage dioxygenase 4 (CCD4) is involved in carotenoid breakdown in senescing leaf and desiccating seed, and is part of the proteome of plastoglobules (PG), which are thylakoid-associated lipid droplets. Here, we demonstrate that CCD4 is functionally active in PG. Leaves of Arabidopsis thaliana ccd4 mutants constitutively expressing CCD4 fused to yellow fluorescent protein showed strong fluorescence in PG and reduced carotenoid levels upon darkinduced senescence. Lipidome-wide analysis indicated that $\beta$-carotene, lutein, and violaxanthin were the principle substrates of CCD4 in vivo and were cleaved in senescing chloroplasts. Moreover, carotenoids were shown to accumulate in PG of ccd4 mutant plants during senescence, indicating translocation of carotenoids to PG prior to degradation.

Keywords: plastoglobule, ccd4, carotenoid, senescence, chloroplast, Arabidopsis thaliana

\section{INTRODUCTION}

Plastoglobules (PG) are chloroplast lipoprotein particles surrounded by a lipid monolayer and associated with the thylakoid membrane via the outer lipid leaflet. PG were first thought only to be lipid storage sites. But a series of studies revealed that PG actively participate in lipid synthesis and repair (Eugeni Piller et al., 2012; Rottet et al., 2015; Spicher and Kessler, 2015). It was shown that PG contain enzymes such as tocopherol cyclase (VTE1), NAD(P)H dehydrogenase C1 (NDC1), and phytyl ester synthase (PES), as well as others (Zbierzak et al., 2010; Eugeni Piller et al., 2011, 2014; Lippold et al., 2012). Subsequently, roles for PG in synthesis and metabolism of plastochromanol, tocopherol, phylloquinone, fatty acid phytyl esters, and triacylglycerol were demonstrated. In chromoplasts, PG accumulate large amounts of carotenoid esters and are implicated in carotenoid biosynthesis. Carotenoid biosynthesis enzymes are recruited to chromoplast PG presumably to channel intermediates and streamline carotenoid production (Ytterberg et al., 2006). A homolog of PES1, pale yellow petal 1 (PYP1) has been implicated in the formation of the abundant carotenoid esters in chromoplasts (Ariizumi et al., 2014). In chloroplasts, carotenoid biosynthetic enzymes associate with membranes rather than PG (Ruiz-Sola and Rodríguez-Concepción, 2012). Little is known about the catabolic fate of photosynthesis-related carotenoids in senescent chloroplasts (Tevini and Steinmüller, 1985; Biswal, 1995). But, it has been shown that carotenoid cleavage dioxygenase 4 (CCD4) is present in the PG proteome (Vidi et al., 2006; Ytterberg et al., 2006; Lundquist et al., 2012) suggesting a role of PG in carotenoid cleavage. 
CCD4 belongs to the carotenoid cleavage dioxygenase family, which has nine members in Arabidopsis thaliana. Across the plant kingdom, CCD4 was shown to be a multifunctional enzyme that carries out a variety of closely related cleavage reactions depending on the developmental stage and tissue (Ohmiya et al., 2006; Rubio et al., 2008; Huang et al., 2009; Ahrazem et al., 2010; Campbell et al., 2010; Rodrigo et al., 2013; Ma et al., 2014; Zhang et al., 2015). In A. thaliana (At) the exact reaction mechanisms remain unsolved. First insight into the biological function of AtCCD4 arose from a PG proteome study, where dark treatment resulted in a twofold accumulation of CCD4 in PG compared to high light treatment (Ytterberg et al., 2006). Therefore, AtCCD4 was predicted to play a role in darkinduced breakdown of carotenoids. Later, it was observed that AtCCD4 was downregulated in the $a b c 1 k 1 a b c 1 k 3$ kinase double mutant, which may explain the increased level of carotenoids measured in PG of $a b c 1 k 1 a b c 1 k 3$ when compared to wild type (WT) (Lundquist et al., 2013). Using linkage mapping and genome-wide association studies, AtCCD4 was identified as a negative regulator of carotenoid content during seed desiccation (Gonzalez-Jorge et al., 2013). The same study also showed that it was implicated in carotenoid breakdown during dark-induced senescence in leaves. In both seed and leaf, $\beta$-carotene was the most affected among a selection of carotenoids (GonzalezJorge et al., 2013). More recently, AtCCD4 was implicated in the formation of different apocarotenoids that serve as signaling molecules (Avendano-Vazquez et al., 2014; Lätari et al., 2015).

This article presents data regarding AtCCD4 localization and function. Co-expression with a fluorescent PG marker protein as well as membrane fractionation, provided strong evidence for AtCCD4 localization in PG. Senescence was induced before lipidome-wide analysis to further characterize the biochemical phenotype of the ccd4 mutants and CCD4 complemented lines. Importantly, the study also demonstrates accumulation of carotenoid substrates in PG of the $c c d 4$ mutant under natural senescence. In summary, the data indicate that PG are a site of carotenoid cleavage adding another function to this chloroplast subcompartment.

\section{RESULTS}

\section{Subcellular Localization of AtCCD4 in Plastoglobules}

CCD4 (At4g19170) encodes a protein of 595 amino acids with a predicted molecular weight of $65.6 \mathrm{kDa}$. A N-terminal chloroplast transit peptide of 34 residues was predicted by ChloroP algorithm ${ }^{1}$. Targeting of CCD4 to the chloroplast and the processing of the predicted transit peptide was assessed in an in vitro import assay using isolated pea chloroplasts (Figure 1A). As demonstrated, pre- $\left[{ }^{35} \mathrm{~S}\right]$ CCD4$6 \mathrm{xHIS}$ was imported and processed inside the chloroplasts to a mature thermolysin-resistant protein of the expected mass.

\footnotetext{
${ }^{1}$ http://www.cbs.dtu.dk/services/ChloroP
}

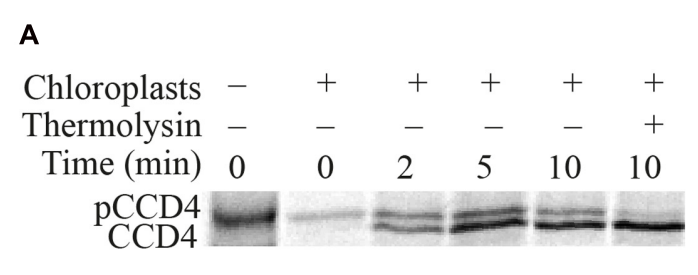

B
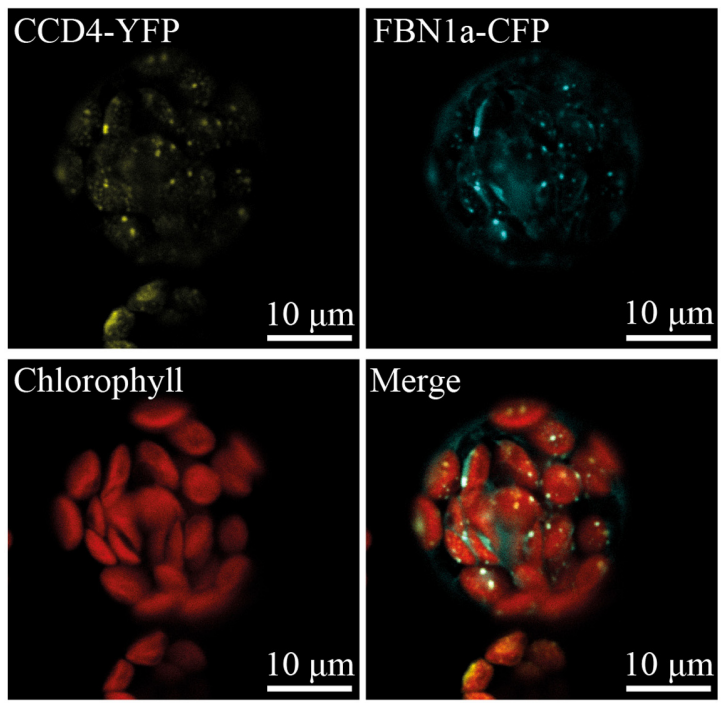

C

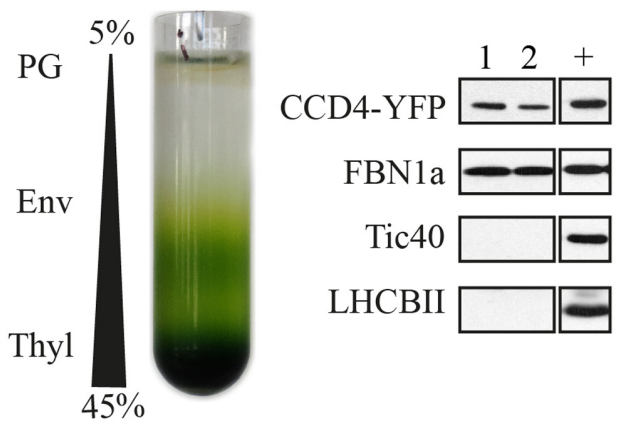

FIGURE 1 | Plastoglobule localization of CCD4. (A) Pre-[ $\left.{ }^{35} \mathrm{~S}\right]$

CCD4-6xHIS (pCCD4) was incubated with isolated Pea chloroplasts from 0 to 10 min in vitro. Upon import, mature [ $\left.{ }^{35} \mathrm{~S}\right] \mathrm{CCD} 4-6 \mathrm{xHIS}$ (CCD4) showed a lower molecular mass due to the removal of the transit peptide and unlike pCCD4 was resistant to thermolysin protease. (B) Colocalization of CCD4-YFP and FBN1a-CFP in isolated protoplasts of Arabidopsis thaliana. (C) Isolation of PG by chloroplast membrane fractionation on a discontinuous sucrose gradient (PG, plastoglobules; Env, envelopes; Thyl, thylakoids). Pure PG fractions (nos. 1 and 2) contain recombinant CCD4-YFP. Tic40, FBN1a and LHCBII are envelope, PG and thylakoid markers, respectively. + , positive control which corresponds to a membrane fraction containing all four markers.

Subcellular localization of CCD4 was analyzed by confocal microscopy of protoplasts prepared from A. thaliana ccd4-2 mutants complemented with CCD4-YFP (yellow fluorescent protein), which were subsequently transformed with a construct encoding FBN1a-CFP (cyan fluorescent protein) 
(Figure 1B). Chloroplasts were identified by their red chlorophyll autofluorescence. CCD4-YFP, as well as the PG marker FBN1aCFP (Vidi et al., 2006), resulted in punctate fluorescence inside the chloroplasts. Moreover, the two colocalized in the fluorescent spots, most likely PG.

To exclude that the fluorescent spots for CCD4-YFP were non-specific protein aggregates, $\mathrm{PG}$ were isolated from transgenic plants by sucrose gradient floatation (Figure 1C). Due to their low density, PG floated to the top of the gradient and were easily isolated by recovering the two first fractions. To evaluate the purity of the PG fractions, the following proteins were used as membrane markers: light-harvesting chlorophyll $a / b$-binding 2 (LHCBII) for thylakoid membranes, translocon at the inner envelope membrane of chloroplasts 40 (Tic40) for the chloroplast envelope membranes, and FBN1a (also known as PGL35) a wellaccepted marker for PG (Lundquist et al., 2012). Pure PG, as supported by the presence of FBN1a and the absence of Tic40 and LHCBII, contained the CCD4-YFP fusion protein (detected by an HA-tag included in the YFP moiety).

\section{Plastoglobules of Senescent ccd4 Contain More $\beta$-Carotene and Lutein}

Because of the localization of CCD4 at PG and the known role of PG in senescence (Besagni and Kessler, 2013; Springer et al., 2015), we investigated the lipid composition of PG during natural senescence in $c c d 4-2$. As a control, the experiment was simultaneously carried out on an unrelated senescing transgenic line. PG were purified by floatation on a discontinuous sucrose gradient using isolated chloroplast membranes (corresponding to $30 \mathrm{mg}$ of chlorophyll). The visible difference between the two PG fractions was striking. The PG fraction from the $c c d 4-2$ mutant appeared orange, while it was normally pale-yellow as seen in the PG fraction from the control line (Figure 2A).

To investigate the composition of $c c d 4-2$ PG, lipid profiling was carried out. Major compounds detected by this method are shown by comparably scaled chromatograms in Figure 2A. The typical PG prenylquinones were observed in both samples, namely $\alpha$-tocopherol, vitamin $\mathrm{K}$, plastochromanol and plastoquinone. Monogalactosyldiacylglycerol (MGDG) 18:3/16:3 appeared as the dominant galactolipid in both PG extracts. Two compounds at $\mathrm{m} / \mathrm{z} 255.2320$ (RT: $1.25 \mathrm{~min}$ ) and $\mathrm{m} / \mathrm{z} 283.2632$ (RT: $1.66 \mathrm{~min}$ ) were tentatively identified as fatty acids 16:0 $\left(\mathrm{C}_{16} \mathrm{H}_{31} \mathrm{O}_{2}\right.$, error $\left.=-1.6 \mathrm{ppm}\right)$ and 18:0 $\left(\mathrm{C}_{18} \mathrm{H}_{35} \mathrm{O}_{2}\right.$, error $=-1.8 \mathrm{ppm})$, respectively. To visualize carotenoids such as $\beta$-carotene and lutein, a magnification of the corresponding chromatogram is shown in the upper left corner.

In order to compare lipid levels in PG, the normalization of PG concentration is critical. We thus considered the total amount of prenylquinones as an internal standard for PG concentration (Lundquist et al., 2013). Relative intensities of $\alpha$-tocopherolquinone, $\alpha-, \delta-, \gamma$-tocopherol, vitamin $\mathrm{K}$, plastochromanol-8 and plastoquinone were added up and used to normalize data. Noteworthy, these prenylquinone contents were not significantly affected by the loss of CCD4 in a whole plant extract. Variation between $c c d 4-2$ and control was obtained by calculating the fold change of lipid normalized intensity. Results are summarized in Table $\mathbf{1}$ and showed a fivefold and a threefold increase in concentration of $\beta$-carotene and lutein, respectively, in $c c d 4-2$. In addition, galactolipids MGDG 18:3/16:3 and 18:3/18:3 were about two to three times more abundant in PG of $c c d 4-2$.

In addition, a spectroscopic analysis was carried out on isolated PG shown in Figure 2A to evaluate the overall carotenoid content by an independent method. The absorption spectra of the PG lipid extracts revealed carotenoid-typical peaks with maxima at 420,450 , and $470 \mathrm{~nm}$ for $c c d 4-2$ which were absent in the control (Figure 2B). Absolute quantification using a commercial $\beta$-carotene standard showed a fivefold higher carotenoid content in $c c d 4-2$ PG compared to that in the control (Figure 2C).

\section{Characterization of CCD4 Complemented Plants}

To investigate CCD4 subcellular localization (shown in Figure 1) and in vivo function, $A$. thaliana $c c d 4-2$ and $c c d 4-4$ mutants (Scholl et al., 2000; Gonzalez-Jorge et al., 2013) were transformed with a construct carrying a glufosinate resistance marker and a fusion between CCD4 and YFP tagged with human influenza hemagglutinin (HA) under control of the constitutive cauliflower mosaic virus $35 \mathrm{~S}$ promoter. Resulting CCD4 complemented plants were $c c d 4-2:: 35 S: C C D 4-Y F P$ (abbreviated as 35S:CCD4.2) and $c c d 4-4:: 35 S: C C D 4-Y F P$ (abbreviated as 35S:CCD4.4). Ten independent glufosinate-resistant $\mathrm{T} 1$ plants were selected based on a visible band at the expected mass of $94.7 \mathrm{kDa}$ by immunoblotting with anti-HA (Figure 3A). T2 progeny of nine lines segregated 3:1 (glufosinate resistant to glufosinate sensitive) indicating a single insertion of the transgene. Among T3 progeny, six independent plants were identified by segregation analysis as being homozygous for the insertion of the 35S:CCD4-YFP construct. CCD4 complemented plants showed a WT phenotype regardless of the $c c d 4$ mutant background. To compare CCD4YFP expression in transgenic plants with that of CCD4 in WT plants we carried out $\mathrm{qPCR}$ reactions. The relative expression of 35S:CCD4.2 and 35S:CCD4.4 were around two and six-times higher than WT (Figure 3D).

Complementation was assessed by phenotypic observation under dark-induced senescence in leaves. Surprisingly, most of the CCD4 complemented lines developed a less yellowish leaf phenotype than WT, suggesting that their carotenoid content was lower. Some lines (e.g., h and j) also showed yellowish leaves similar to the corresponding $c c d 4-4$ mutant (Figure 3B). However, in most transgenic lines CCD4-YFP appeared to complement $c c d 4$, which is evident from the lack of yellowing of the leaves upon senescence. Note that chlorophyll distribution seemed irregularly affected in both $c c d 4$ and CCD4 complemented lines (Figure 3B).

To quantify the visual observations, total chlorophyll and carotenoids were rapidly extracted and analyzed by spectrophotometry (Figure 3C). As expected, the CCD4 complemented lines contained significantly $(P<0.001)$ less carotenoids than the WT. In contrast, $c c d 4$ contained significantly $(P<0.05)$ more carotenoids than the WT. Chlorophyll contents showed no statistical difference among the genotypes. In conclusion, the constitutive expression of 
A
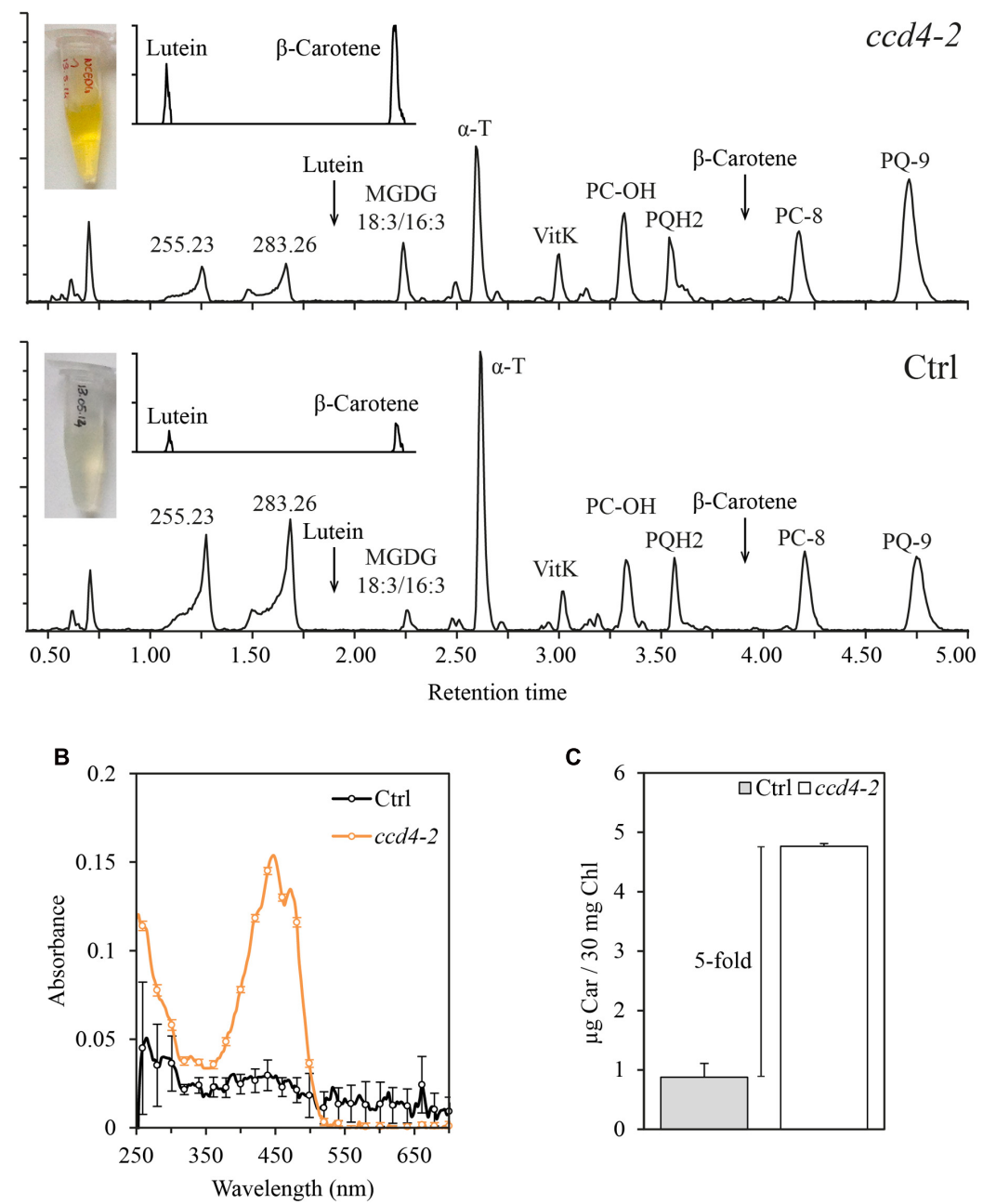

FIGURE 2 | Carotenoids accumulated in senescent plastoglobules of ccd4-2. (A) PG were isolated from naturally senescent ccd4-2 and control plants (3 months old) by a sucrose gradient loaded with a chlorophyll-equivalent of $30 \mathrm{mg}$ of membranes. Lipids from the PG fractions were analyzed by untargeted ultra-high pressure liquid chromatography-atmospheric pressure chemical ionization-quadrupole time of flight mass spectrometry and chromatograms of detected lipids are shown. (B) Spectrophotometric analysis of PG fractions. Three maxima around $450 \mathrm{~nm}$, typical of carotenoid absorption spectra, were detected in ccd4-2 $P G$. (C) Quantification of total carotenoid contents in PG fractions. Absorbance at $448 \mathrm{~nm}$ was recorded and compared with a calibration curve of $\beta$-carotene standard. Carotenoid content is expressed in $\mu \mathrm{g}$ of $\beta$-carotene per $30 \mathrm{mg}$ of chlorophyll of total membranes used in the experiment. MGDG, monogalactosyldiacylglycerol; $\alpha$-T, $\alpha$-tocopherol; VitK, vitamin K; PC-OH, hydroxy-plastochromanol; PQH2, plastoquinol; PC-8, plastochromanol; PQ-9, plastoquinone; Ctrl, control; Car, $\beta$-carotene; Chl, chlorophyll.

CCD4-YFP did not cause an apparent phenotype under standard growth conditions. However, the complemented plants exhibited elevated carotenoid degradation under dark-induced senescence, as supported by less yellowish leaves and reduced total carotenoid contents.

\section{Enhanced Carotenoid Degradation in CCD4 Complemented Plants}

To assess the effects of constitutive CCD4-YFP expression on the degradation of carotenoids, we investigated the lipid contents in leaves before and after dark-induced senescence of CCD4 complemented A. thaliana plants. Data obtained from untargeted ultra-high pressure liquid chromatographyatmospheric pressure chemical ionization-quadrupole time of flight mass spectrometry (UHPLC-APCI-QTOFMS) was subjected to partial least squares-discriminant analysis (PLS-DA) in order to investigate significant differences between treatments. Our findings underlined an absence of statistical difference between WT and CCD4 complemented plants under standard growth conditions, whereas senescence resulted in genotypeclustering with the first component explaining $26.9 \%$ of the variability (Figure 4A). Under senescence conditions, the PLSDA model was validated with a permutation test $(n=200$; $\left.Q_{2}=0.0,-0.218\right)$. As expected, 35S:CCD4.2 and 35S:CCD4.4 displayed a similar lipid profile, which was different from that 
TABLE 1 | Lipid content of senescent ccd4-2 PG compared to control.

\begin{tabular}{lc}
\hline PG lipid & Fold change \\
\hline$\beta$-Carotene & 5.2 \\
MGDG-18:3/16:3 & 3.3 \\
Lutein & 3.2 \\
MGDG-18:3/18:3 & 2.1 \\
Plastoquinone & 2.0 \\
Vitamin K & 1.4 \\
Plastoquinol & 1.1 \\
Plastochromanol & 1.1 \\
$\alpha$-Tocopherol-quinone & -1.0 \\
DGDG-18:1/18:3 & -1.2 \\
MGDG-18:2/18:3 & -1.4 \\
$\alpha$-Tocopherol & -1.5 \\
$\delta$-Tocopherol & -1.6 \\
$\gamma$-Tocopherol & -1.8 \\
\hline
\end{tabular}

Relative peak intensity from chromatograms shown in Figure $2 A$ was normalized for total prenylquinones prior to calculating the fold change between ccd4-2 and control.

of WT. The loading plot was investigated to point out the main compounds responsible for the discrimination between WT and CCD4 complemented lines. Among more than 300 lipids measured, the few outlying compounds were identified whenever possible (Figure 4B) and relative quantifications subjected to Student's $t$-test. In brief, WT accumulated significantly more lutein and $\beta$-carotene than CCD4 complemented plants. What is more, an unidentified compound at $\mathrm{m} / \mathrm{z} 400.3339$ (RT: $2.41 \mathrm{~min}$, $\mathrm{C}_{27} \mathrm{H}_{44} \mathrm{O}_{2}$ ) was three times higher in WT. Polar lipids such as MGDG 18:3/16:3, 18:3/18:3, DGDG 18:3/18:3, and PE 18:2/16:0 were more abundant in CCD4 complemented plants.

The carotenoid-targeted UHPLC analysis enabled an absolute and accurate quantification of carotenoids (Figure 5). On the whole, total carotenoid levels in senescent leaves of CCD4 complemented plants (63.7 $\mu \mathrm{g} \mathrm{g}^{-1} \mathrm{FW}$ (fresh weight)) were 1.8 times lower than those of the WT (113.57 $\left.\mu \mathrm{g} \mathrm{g}^{-1} \mathrm{FW}\right)$ and 2.3 times lower than those of $c c d 4\left(149.4 \mu \mathrm{g} \mathrm{g}^{-1} \mathrm{FW}\right)$. The contents of $\beta$-carotene, lutein and violaxanthin were all significantly reduced in CCD4 complemented plants while neoxanthin was not. Neoxanthin and violaxanthin contents were the least affected in the $c c d 4$ mutant. In contrast, violaxanthin was reduced about threefold in CCD4 complemented plants compared with WT.

\section{DISCUSSION}

CCD4 has a predicted cleavable N-terminal chloroplast transit peptide. In agreement with bioinformatics predictions and a previous study (Naested et al., 2004), CCD4 was successfully imported into isolated chloroplasts demonstrating chloroplast localization (Figure 1). The association of CCD4 with PG was addressed using two independent approaches: (a) colocalization with a known PG marker and (b) subfractionation of chloroplasts. Both approaches provided clear evidence for association of CCD4 with PG (Figure 1). The association of
CCD4 with PG provides the molecular framework for a role of this chloroplast subcompartment in carotenoid cleavage.

The localization of CCD4 in PG suggests that during senescence carotenoids move to PG to be cleaved. Therefore accumulation of uncleaved carotenoids in $c c d 4$ mutant PG may be expected. We used a lipidomics approach to address the role of CCD4 and PG in carotenoid breakdown. For this purpose, PG from $c c d 4$ mutant and control plants were analyzed. Even visually, ccd4 PG fractions were distinctly more orange than the control (Figure 2A). This observation was confirmed by untargeted lipid analysis demonstrating that they contained fivefold and threefold more $\beta$-carotene and lutein, respectively (Table 1). $\mathrm{ABC} 1 \mathrm{~K} 1$ and $-\mathrm{K} 3$ are regulatory kinases associating with PG (Lundquist et al., 2013; Martinis et al., 2013, 2014). In the corresponding double mutant, the CCD4 gene was downregulated and PG contained higher levels of carotenoids than the WT (Lundquist et al., 2013). These earlier findings provide additional evidence for the role of CCD4 and PG in carotenoid cleavage.

Our observations are in agreement to Gonzalez-Jorge et al. (2013), which reported CCD4 as being a major negative regulator of $\beta$-carotene in senescent leaves. Here, constitutive overexpression of CCD4 in the $c c d 4$ mutant background increased degradation of carotenoids upon senescence (Figure 3) but under standard growth conditions the carotenoid content was indistinguishable from the WT. This observation may be explained in the following way: in vegetative chloroplasts, carotenoids associate with the light-harvesting complex (LHC) proteins in the thylakoids (Croce et al., 1999; Li et al., 2000; Dall'Osto et al., 2010). Under these conditions carotenoids are stably bound and inaccessible to CCD4. Only upon senescence would LHC be proteolytically degraded. The carotenoids are then free to diffuse to PG for CCD4-dependent cleavage.

Interestingly, the untargeted lipid analysis of senescing overexpressing CCD4 plants also indicated higher levels of galacto- and phospholipids than in the WT (Figure 4). These lipids are major constituents of the thylakoid membrane. It is therefore possible that the degradation of thylakoid membranes in CCD4 overexpressing plants is retarded under senescence conditions. Potentially, CCD4-dependent carotenoid cleavage products, commonly termed apocarotenoids, may serve as signal ensuring the synchronization of membrane breakdownrelated processes (Auldridge et al., 2006; Walter et al., 2010). For instance, AtCCD4 was implicated in the formation of an unknown apocarotenoid that serves as a signaling molecule in zeta-carotene desaturase ( $z d s$ ), an albino mutant arrested in early chloroplast development and defective in carotenoid synthesis (unable to convert $\zeta$-carotene into lycopene) (AvendanoVazquez et al., 2014). Moreover, AtCCD4 was correlated with the formation of $\mathrm{C}_{13}$-apocarotenoid glycosides that occur in overexpressors of phytoene synthase (PSY), which catalyzes the rate-limiting step in carotenoid biosynthesis (Lätari et al., 2015). Taken together, these results suggest that CCD4 acts on carotenoid turnover in leaves of $A$. thaliana producing apocarotenoids that may be metabolized further. Through this activity, a role of CCD4 in the production of retrograde signaling 


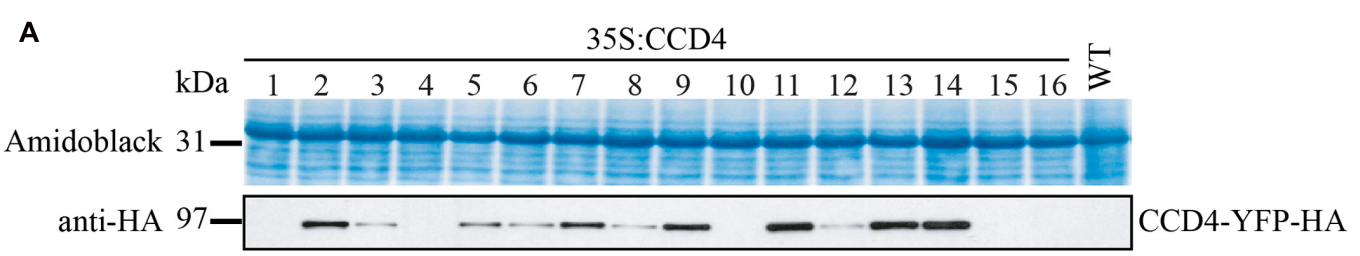

B
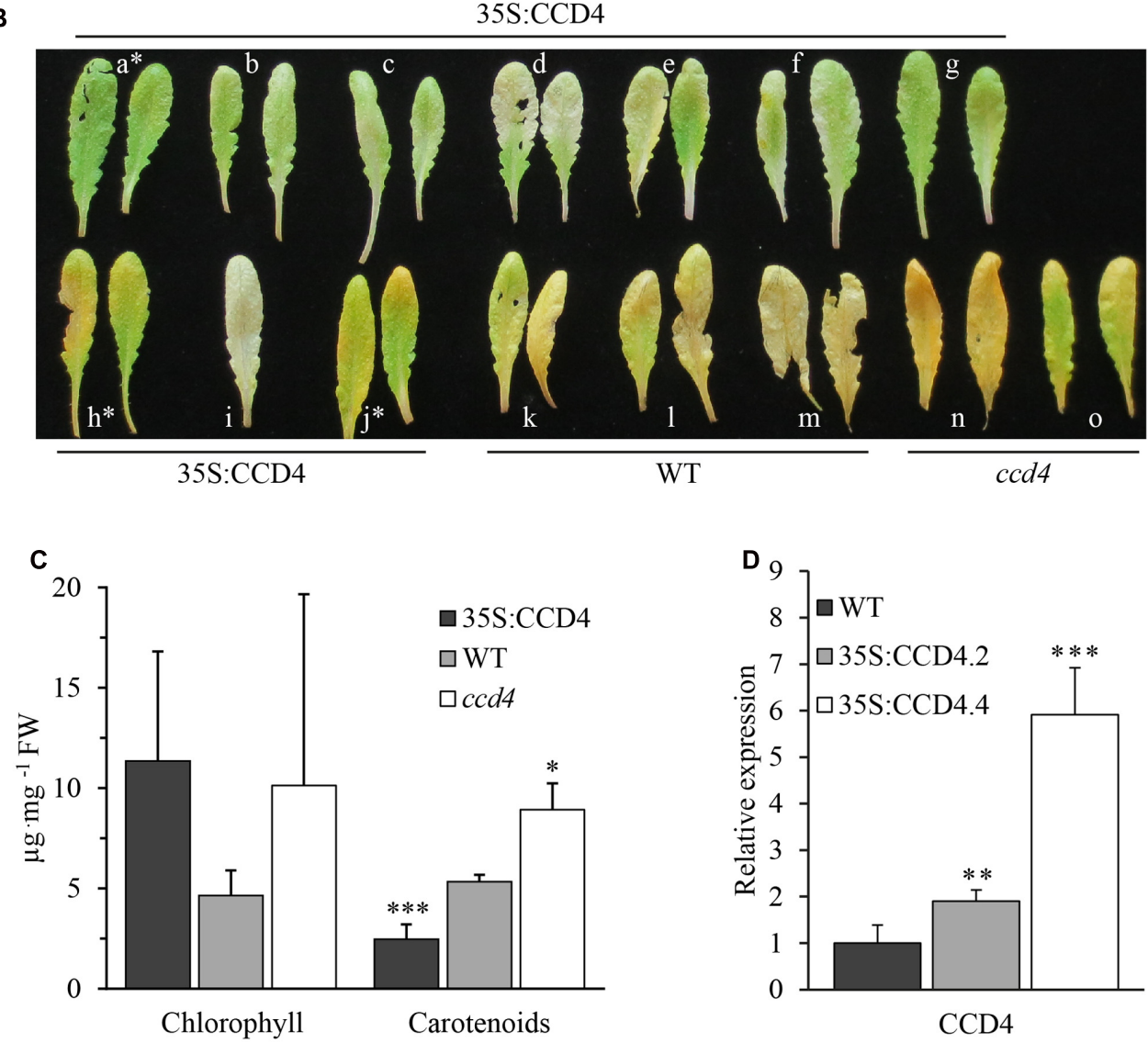

FIGURE 3 | Complementation of ccd4 mutants with 35S:CCD4-YFP-HA. (A) Relative amounts of CCD4-YFP-HA in complemented lines were assessed by immunoblot analysis with anti-HA antibodies. A representative blot of 16 independent lines is shown, none of which showed a visible phenotype.

(B) Complementation analysis of CCD4 complemented lines by dark-induced senescence. Detached leaves were subjected to dark-induced senescence for 10 days and complementation was determined based on the phenotype. Two 35S:CCD4 plants (h and j) were not complemented as they showed a yellowish color similar to ccd4-4. (C) Total chlorophyll and carotenoids were extracted from the leaves shown in (B) (NB: the white asterisks mark the leaves that were excluded for the calculations). Concentrations of pigments were established with a spectrophotometer. Means \pm SD were calculated for CCD4 complemented plants $(n=7)$, WT $(n=3)$ and ccd4 $(n=2)$. Statistical significance was determined by a Student's $t$-test (unpaired homoscedastic, two-tailed, $\left.{ }^{*} P<0.05,{ }^{* * *} P<0.001\right)$. (D) CCD4 gene expression analysis. Transcripts of CCD4 were quantified by qPCR in the WT and in the two complemented lines, i.e., 35S:CCD4.2 and 35S:CCD4.4. The relative expression of CCD4 was normalized to the reference gene ACTIN. Means $\pm \operatorname{SD}(n=6)$. Statistical significance between transgenic lines and the WT was determined by a Student's $t$-test (unpaired heteroscedastic, two-tailed, ${ }^{* *} P<0.01,{ }^{* * *} P<0.001$ ).

molecules that relay information on the chloroplast state for downstream gene regulation appears likely.

Untargeted as well as targeted analyses identified lutein, $\beta$-carotene and violaxanthin as the most important targets of CCD4 during senescence (Figures 4 and 5). When comparing all genotypes, the carotenoids that accumulated in $c c d 4$ were cleaved more extensively in CCD4 overexpressing plants than in the WT providing additional evidence for the role of CCD4
(Figure 5). However, our analysis did not identify a distinct carotenoid cleavage product. This suggests that cleavage may result in a variety of products including volatiles that may escape identification by liquid chromatography-mass spectrometry. The data in this paper indicate PG as a site of carotenoid-cleavage in senescent chloroplasts. In the future it will be of interest to identify the cleavage products and their potential functions as signaling molecules. 


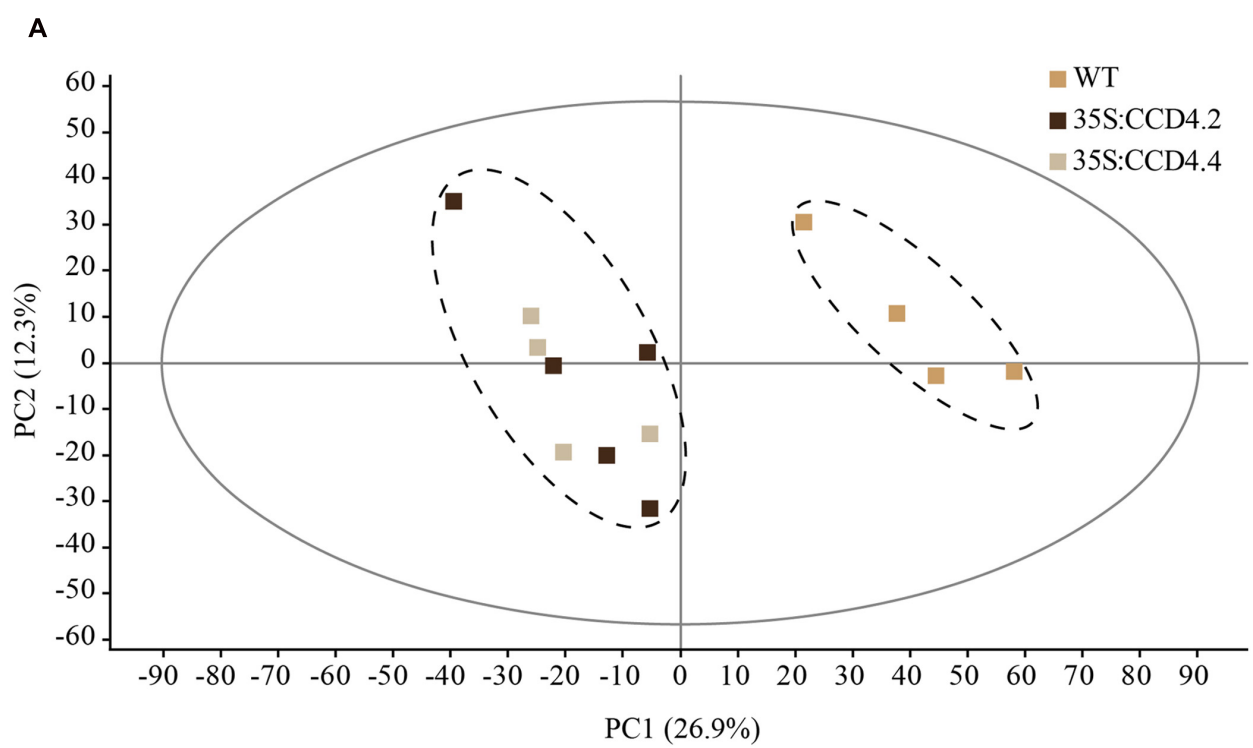

B

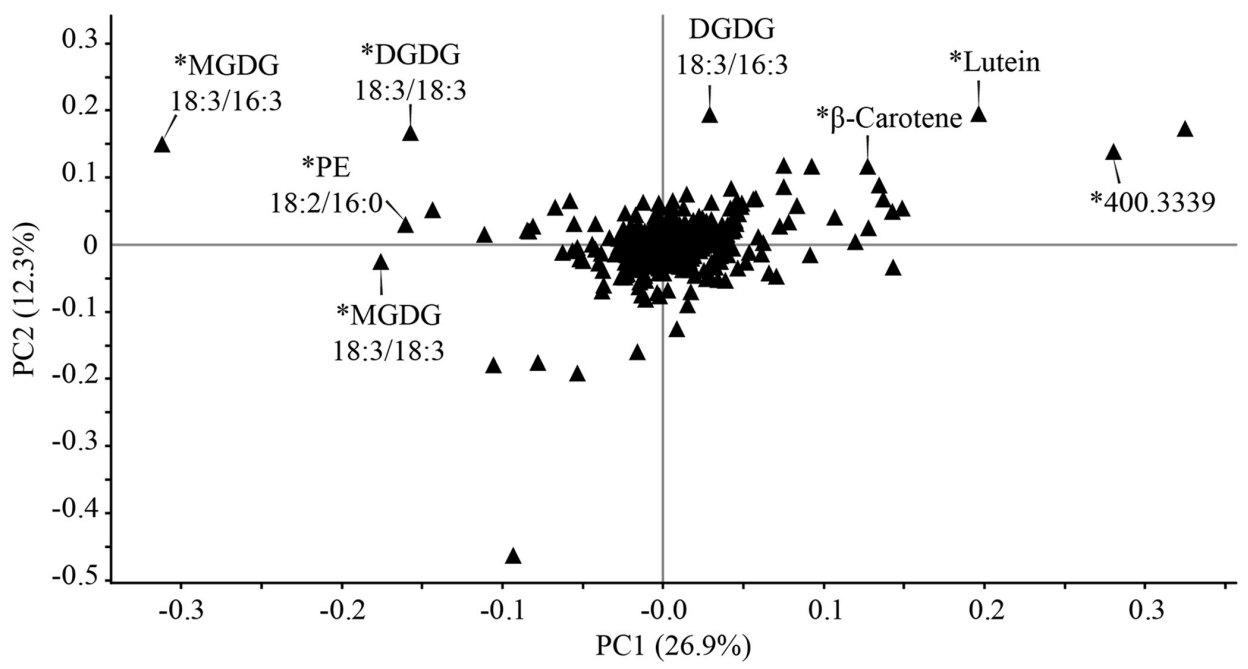

FIGURE 4 | Constitutive expression of CCD4 resulted in a distinct lipid profile during dark-induced senescence in leaves when compared with WT.

(A) Detached leaves were placed in darkness for 8 days prior to lipid extraction $(n=5)$. Lipids were analyzed by ultra-high pressure liquid chromatography-atmospheric pressure chemical ionization-quadrupole time of flight mass spectrometry. Discrimination was evaluated with a partial least squares-discriminant analysis in which outlying samples were removed prior to data processing. (B) Compounds responsible for the difference between CCD4 complemented lines and WT are indicated on the loading plot. *Statistically significant according to Student's t-test (unpaired heteroscedastic, two-tailed). PC, principal component.

\section{MATERIALS AND METHODS}

\section{Plant Material and Growth Conditions}

Arabidopsis WT was Columbia-0. Two T-DNA insertion mutants from A. thaliana, ccd4-2 (Salk_097984) and $c c d 4$ 4 (Salk_010751), disrupted in CCD4 (At4g19170) gene were obtained from the European Arabidopsis Stock Centre (Scholl et al., 2000). Seeds were surface sterilized $(0.05 \% \mathrm{v} / \mathrm{v}$ Triton $\mathrm{X}-100,70 \% \mathrm{v} / \mathrm{v}$ ethanol) and sowed on solidified $1 / 2 \mathrm{MS}$ (Murashige and Skoog) medium (Murashige and Skoog, 1962).
Germination was induced and synchronized at $4^{\circ} \mathrm{C}$ for 2 days. Plants were grown under short day conditions $(8 \mathrm{~h}$ light, $16 \mathrm{~h}$ dark, $150 \mu \mathrm{mol} \mathrm{m}^{-2} \mathrm{~s}^{-1}$ ) in a GroBank system (CLF Plant Climatics). Afterward, plants were further grown on soil (Ricoter). Alternatively, seeds were directly sowed on soil.

Senescence was induced by detaching the whole rosette from roots and placing it on a filter paper slightly humidified (tap water) in a Petri dish for 7 days in the dark. Alternatively, leaves were detached from the whole rosette and subjected to dark treatment as per described. 

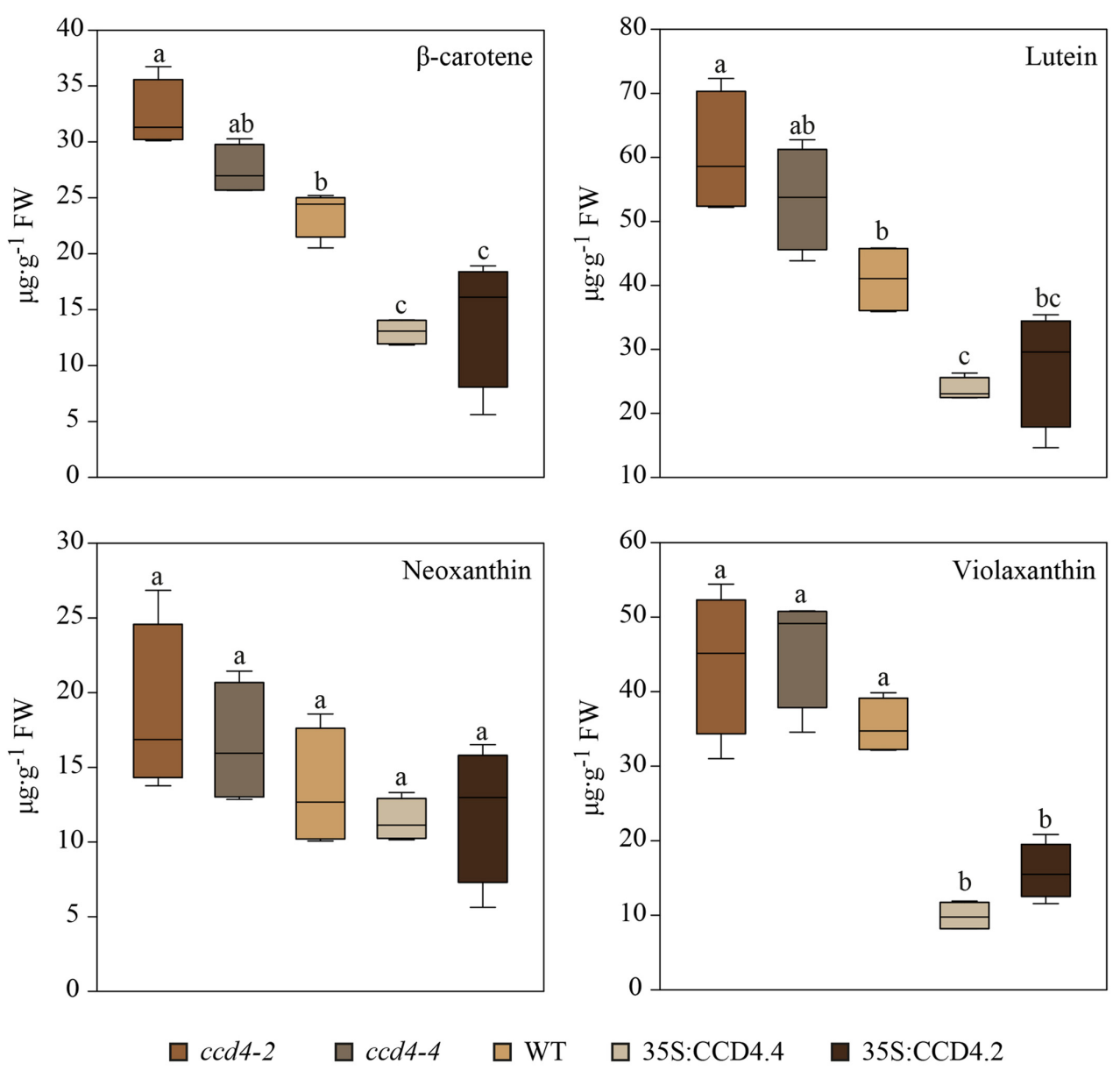

FIGURE 5 | Reduced level of photosynthesis-related carotenoids during senescence in CCD4 complemented plants. Detached leaves were placed in darkness for 8 days prior to lipid extraction $(n=5)$. Lipids were separated by UHPLC-DAD following the carotenoid-profiling protocol. Absolute quantification of carotenoids was carried out on the basis of calibration curves obtained with pure standards. Box plots created with SigmaPlot are shown. Statistical differences across genotypes were assessed with a one-way ANOVA. Pairwise multiple comparisons (Holm-Sidak method) revealed significant differences (letters are distributed accordingly, $P<0.05)$.

\section{Plasmid Construction}

Genomic DNA from A. thaliana was used to amplify CCD4 gene by PCR using the primer couple CCD4TopoF (5'CACCATGGACTCTGTTTCTTCTTCTTCC-3') and CCD4T opoR ( $5^{\prime}$-CCATGGAAGCTTATTAAGGTCACTTTCC- $\left.3^{\prime}\right)$. The PCR product was inserted into the pENTR D-TOPO entry vector using the Gateway ${ }^{\circledR}$ BP Clonase ${ }^{\circledR}$ II Enzyme Mix (Invitrogen). CCD4 was then recombined from the entry vector to two other Gateway-based destination vectors (Gateway ${ }^{\circledR}$ LR Clonase ${ }^{\circledR}$ II Enzyme Mix, Invitrogen), namely p0GWA bacterial expression vector (Busso et al., 2005) and pEarleyGate101 plant expression vector (Earley et al., 2006). Respectively, the constructs obtained were T7:CCD4-6xHIS and 35S:CCD4-YFP-HA.

\section{Complementation of $c c d 4$ with 35S:CCD4-YFP-HA}

The pEarlyGate101-CCD4 construct was introduced by electroporation into the Agrobacterium tumefaciens C58 strain. Flowering $A$. thaliana of $c c d 4$ ( $c c d 4-2$ and $c c d 4-4)$ was transformed with pEarlyGate101-CCD4 construct using the floral dip method as described (Clough and Bent, 1998). Complemented plants were named 35S:CCD4.2 or 35S:CCD4.4 according to the mutant background in which the transgene was expressed (ccd4-2 or $c c d 4-4$, respectively). Transgenic plants were selected on solidified $1 / 2$ MS medium supplemented with $30 \mathrm{mg} / \mathrm{L}$ of glufosinate ammonium. Segregation analyses were carried out to select lines with a single insertion of the transgene.

\section{RNA Extraction, cDNA Synthesis, and qPCR}

RNA was isolated from 2-week-old plants grown on $1 / 2$ MS medium (supplemented with $30 \mathrm{mg} \mathrm{L}^{-1}$ of glufosinate ammonium for transgenic lines) using the RNeasy Plant Mini Kit from Qiagen, which included a DNase treatment. $1 \mu \mathrm{g}$ of RNA was used for reverse transcription with the GoScript ${ }^{\text {TM }}$ Reverse Transcription System (Promega). qPCR was performed 
with FastStart Essential DNA Green Master on the LightCycler from Roche. Primers for actin were obtained from Qiagen (Act2 QT00774634) and those for CCD4 were designed as published elsewhere (Gonzalez-Jorge et al., 2013).

\section{Transformed Protoplasts Observed by Confocal Microscopy}

Plants were grown for 3 weeks on $1 / 2$ MS supplemented with $1 \%$ sucrose. For colocalization with CCD4-YFP, the vector pCL62 carrying FBN1a in frame with CFP was used (Vidi et al., 2006). Protoplasts were isolated and transformed as described (Jin et al., 2001). Fluorescence in protoplasts was monitored $30 \mathrm{~h}$ after transformation with a Leica TCS SP5 (Leica Microsystems) confocal microscope using the appropriate parameters for YFP, CFP and chlorophyll autofluorescence. The YFP was excited by the 514-nm Argon laser line with 525-600-nm detection windows. CFP fluorescence was detected using the 458-nm Argon laser line and 465-505-nm detection windows. Settings were checked with untransformed protoplasts for one and both constructs to avoid unspecific background noise.

\section{Isolation of PG on a Sucrose Gradient}

Intact chloroplasts from 8-week-old plants were isolated essentially as described (Hiltbrunner et al., 2001; Kessler and Glauser, 2014). Membrane fractionation, verification by SDSPAGE and immunoblotting were adapted from published protocols (Vidi et al., 2006; Besagni et al., 2011; Kessler and Glauser, 2014). Lysed and homogenized chloroplasts were subjected to $1 \mathrm{~h}$ of ultracentrifugation (Optima XPN80 Ultracentrifuge, Beckman Coulter) to separate the total membranes from the stroma. Subsequently, homogenized membranes (corresponding to $3.3 \mathrm{mg}$ of chlorophyll) were separated with a miniaturized discontinuous sucrose gradient as follows. On the top of the $2 \mathrm{~mL}$ membrane-containing $45 \%$ sucrose, $804 \mu \mathrm{L}$ of $38 \%, 804 \mu \mathrm{L}$ of $20 \%, 539 \mu \mathrm{L}$ of $15 \%$, and $845 \mu \mathrm{L}$ of $5 \%$ sucrose were layered in a $5 \mathrm{~mL}$ Ultra-Clear ${ }^{\mathrm{TM}}$ Centrifuge Tube $(13 \mathrm{~mm} \times 51 \mathrm{~mm}$, Beckman Coulter). Gradients were centrifuged at 100,000 $\times g$ for $15 \mathrm{~h}$ in a SW55Ti swinging-bucket rotor (Beckman Coulter). Fractions of $200 \mu \mathrm{L}$ were collected from the top (no. 1) to the bottom of the gradient (no. 25) with a micropipette.

\section{Pea Chloroplast Isolation and Protein Import}

Intact chloroplasts were isolated from 2- to 3-week-old peas (Pisum sativum) as described in Smith et al. (2003) with minor modifications. In vitro translated CCD4 was produced from the p0GWA-CCD4 construct with the $\mathrm{TNT}^{\circledR}$ Quick Coupled Transcription/Translation System (Promega) following the manufacturer's recommendations. The ${ }^{35} \mathrm{~S}$-radiolabeled CCD4-6xHIS was incubated with isolated import competent pea chloroplasts according to the protocol described in Smith et al. (2003).

\section{Lipid Extraction from PG for Untargeted UHPLC Analysis}

The extraction of lipids from isolated PG was carried out as described (Eugeni Piller et al., 2014; Kessler and Glauser, 2014) with some modifications. To remove sucrose, $400 \mu \mathrm{L}$ of fraction were partitioned two times against ethyl acetate (1:1). The upper phases were collected, combined, and evaporated. Pellets of lipids were dissolved in $100 \mu \mathrm{L}$ of tetrahydrofuran for UHPLC-APCIQTOFMS analysis.

\section{Total Carotenoid Quantification in PG by Spectrophotometry}

From a sucrose-gradient loaded with a chlorophyll-equivalent of $30 \mathrm{mg}$ of membranes (Vidi et al., 2006), $30 \mu \mathrm{L}$ of the PG fraction was mixed with $100 \mu \mathrm{L}$ of ethyl acetate. After brief centrifugation in a microfuge, $90 \mu \mathrm{L}$ of supernatant was collected and evaporated. The pellet was resuspended in $10 \mu \mathrm{L}$ of ethyl acetate and the visible spectrum was measured using a Nanodrop 1000 Spectrophotometer (Thermo Fisher Scientific). A calibration curve was established in ethyl acetate using $\beta$-carotene as the standard. The carotenoid content was determined as the absorbance at $448 \mathrm{~nm}$ in ethyl acetate and is expressed as micrograms of $\beta$-carotene equivalent per total membrane loaded, which is expressed in milligrams of chlorophyll ( $\mu \mathrm{g}$ Car/30 mg of chlorophyll).

\section{Pigment Quantification in Senescent Leaves by Spectrophotometry}

Pigments were extracted from leaves using dimethylformamide (DMF). Leaves (50 mg FW) were finely ground in a microcentrifuge tube using a metallic pestle under liquid nitrogen, after which five volumes of DMF and 5-10 glass beads (Assistant, Sontheim, Germany) were added. Samples were homogenized for $3 \mathrm{~min}$ at $30 \mathrm{~Hz}$ in a tissue lyser (Retsch MM 300, Haan, Germany). Then, tubes were centrifuged $3 \mathrm{~min}$ at $16,000 \times g$. Finally, $2 \mu \mathrm{L}$ of supernatant were analyzed with a Nanodrop 1000 Spectrophotometer (Thermo Fisher Scientific). Absorbance was recorded at the following four wavelengths: $480,647,652$, and $664 \mathrm{~nm}$. To determine concentrations of total carotenoids, we applied published equations (Wellburn, 1994). Total chlorophyll was estimated by dividing the absorbance measured at $652 \mathrm{~nm}$ by the factor 36 .

\section{Carotenoid Extraction for Targeted UHPLC Analysis}

To protect carotenoids from oxidation, the following procedures were carried out in semi darkness. Leaves or rosette tissues (100 mg FW) were ground in a cold mortar in presence of liquid nitrogen, and immediately transferred to a microcentrifuge tube. Five volumes of tetrahydrofuran-methanol $(50: 50, v / v)$ and 5-10 glass beads (Assistant, Sontheim, Germany) were added. Samples were further homogenized for $3 \mathrm{~min}$ at $30 \mathrm{~Hz}$ in a tissue lyser (Retsch MM 300, Haan, Germany). Tubes were centrifuged 5 min at $16,000 \times g$ (Eppendorf Centrifuge 5415D) and $400 \mu \mathrm{L}$ of supernatant was transferred to another $1.5 \mathrm{~mL}$ microcentrifuge tube. Centrifugation was repeated and $150 \mu \mathrm{L}$ of supernatant was 
transferred to an amber glass vial (BGB, Boeckten, Switzerland) for UHPLC analysis. Samples were either directly analyzed or stored at $-80^{\circ} \mathrm{C}$ for up to 1 week.

Carotenoid quantification was achieved using UHPLC-DAD (diode array detector). The separation was carried out on a Waters Acquity BEH C18 column $(50 \mathrm{~mm} \times 2.1 \mathrm{~mm}, 1.7 \mu \mathrm{m}$ particle size) held at $50^{\circ} \mathrm{C}$ at a flow rate of $0.7 \mathrm{~mL} \mathrm{~min}{ }^{-1}$. The following gradient program using water as solvent $\mathrm{A}$ and acetonitrile as solvent B was employed: $65-80 \%$ B for $5 \mathrm{~min}, 80-$ $100 \%$ B for $1 \mathrm{~min}$, holding at $100 \%$ B for $3.5 \mathrm{~min}$, reequilibration at $65 \% \mathrm{~B}$ for $1 \mathrm{~min}$. Total analysis time was $10.5 \mathrm{~min}$. A volume of $2.5 \mu \mathrm{L}$ was injected. For quantification, the UV trace at $450 \mathrm{~nm}$ was used. Calibration solutions were prepared as follows: Lutein, violaxanthin, neoxanthin and zeaxanthin were mixed and diluted at $20,5,2$, and $0.5 \mu \mathrm{g} \mathrm{mL}^{-1}$ in tetrahydrofuran-methanolwater $(42.5: 42.5: 15, \mathrm{v} / \mathrm{v})$. Since $\beta$-carotene is highly apolar, it was separately prepared in tetrahydrofuran-water $(85: 15, \mathrm{v} / \mathrm{v})$ at 20 , 5,2 , and $0.5 \mu \mathrm{g} \mathrm{mL}^{-1}$.

\section{Lipid Extraction from Whole Plant for Untargeted UHPLC Analysis}

For untargeted lipid profiling, pure tetrahydrofuran was used for extraction instead of tetrahydrofuran-methanol. Lipid profiling was performed using a UHPLC-APCI-QTOFMS (Waters) as previously described (Martinis et al., 2011; Kessler and Glauser,

\section{REFERENCES}

Ahrazem, O., Trapero, A., Gomez, M. D., Rubio-Moraga, A., and GomezGomez, L. (2010). Genomic analysis and gene structure of the plant carotenoid dioxygenase 4 family: a deeper study in Crocus sativus and its allies. Genomics 96, 239-250. doi: 10.1016/j.ygeno.2010.07.003

Ariizumi, T., Kishimoto, S., Kakami, R., Maoka, T., Hirakawa, H., Suzuki, Y., et al. (2014). Identification of the carotenoid modifying gene PALE YELLOW PETAL 1 as an essential factor in xanthophyll esterification and yellow flower pigmentation in tomato (Solanum lycopersicum). Plant J. 79, 453-465. doi: $10.1111 /$ tpj. 12570

Auldridge, M. E., McCarty, D. R., and Klee, H. J. (2006). Plant carotenoid cleavage oxygenases and their apocarotenoid products. Curr. Opin. Plant Biol. 9, 315-321. doi: 10.1016/j.pbi.2006.03.005

Avendano-Vazquez, A. O., Cordoba, E., Llamas, E., San Roman, C., Nisar, N., De la Torre, S., et al. (2014). An uncharacterized apocarotenoid-derived signal generated in zeta-carotene desaturase mutants regulates leaf development and the expression of chloroplast and nuclear genes in Arabidopsis. Plant Cell 26, 2524-2537. doi: 10.1105/tpc.114.123349

Besagni, C., and Kessler, F. (2013). A mechanism implicating plastoglobules in thylakoid disassembly during senescence and nitrogen starvation. Planta 237, 463-470. doi: 10.1007/s00425-012-1813-9

Besagni, C., Piller, L. E., and Bréhélin, C. (2011). Preparation of plastoglobules from Arabidopsis plastids for proteomic analysis and other studies. Methods Mol. Biol. 775, 223-239. doi: 10.1007/978-1-61779-237-3_12

Biswal, B. (1995). Carotenoid catabolism during leaf senescence and its control by light. J. Photochem. Photobiol. B Biol. 30, 3-13. doi: 10.1016/1011-1344(95) 07197-A

Busso, D., Delagoutte-Busso, B., and Moras, D. (2005). Construction of a set Gateway-based destination vectors for high-throughput cloning and expression screening in Escherichia coli. Anal. Biochem. 343, 313-321. doi: 10.1016/j.ab. 2005.05.015

Campbell, R., Ducreux, L. J. M., Morris, W. L., Morris, J. A., Suttle, J. C., Ramsay, G., et al. (2010). The metabolic and developmental roles of carotenoid
2014). Raw data was processed using Markerlynx $X^{\mathrm{TM}}$ (Waters) for automatic peak detection.

\section{AUTHOR CONTRIBUTIONS}

FK conceived research plans; $\mathrm{CB}$ and FK supervised the experiments; SR and JD performed experiments with the technical assistance of VD; GG carried out lipidomics; SR designed the experiments and analyzed the data; and SR wrote the article with contributions from all of the authors.

\section{FUNDING}

The authors acknowledge the University of Neuchâtel and Swiss National Science Foundation (Grants 31003A_156998 and IZEBZ0_143169).

\section{ACKNOWLEDGMENTS}

The authors wish to thank Claire Bréhélin and Angélica Lima for engineering the pEarlyGate101-CCD4 and p0GWA-CCD4 constructs, and for carrying out preliminary experiments. Shoko Okada and Jason Grant are gratefully acknowledged for their critical reading of the manuscript.

cleavage dioxygenase 4 from potato. Plant Physiol. 154, 656-664. doi: 10.1104/ pp.110.158733

Clough, S. J., and Bent, A. F. (1998). Floral dip: a simplified method for Agrobacterium-mediated transformation of Arabidopsis thaliana. Plant J. 16, 735-743. doi: 10.1046/j.1365-313X.1998. 00343.x

Croce, R., Weiss, S., and Bassi, R. (1999). Carotenoid-binding sites of the major light-harvesting complex II of higher plants. J. Biol. Chem. 274, 29613-29623. doi: 10.1074/jbc.274.42.29613

Dall'Osto, L., Cazzaniga, S., Havaux, M., and Bassi, R. (2010). Enhanced photoprotection by protein-bound vs free xanthophyll pools: a comparative analysis of chlorophyll b and xanthophyll biosynthesis mutants. Mol. Plant 3, 576-593. doi: 10.1093/mp/ssp117

Earley, K. W., Haag, J. R., Pontes, O., Opper, K., Juehne, T., Song, K., et al. (2006). Gateway-compatible vectors for plant functional genomics and proteomics. Plant J. 45, 616-629. doi: 10.1111/j.1365-313X.2005.02617.x

Eugeni Piller, L., Abraham, M., Dörmann, P., Kessler, F., and Besagni, C. (2012). Plastid lipid droplets at the crossroads of prenylquinone metabolism. J. Exp. Bot. 63, 1609-1618. doi: 10.1093/jxb/ers016

Eugeni Piller, L., Besagni, C., Ksas, B., Rumeau, D., Bréhélin, C., Glauser, G., et al. (2011). Chloroplast lipid droplet type II NAD(P)H quinone oxidoreductase is essential for prenylquinone metabolism and vitamin K1 accumulation. Proc. Natl. Acad. Sci. U.S.A. 108, 14354-14359. doi: 10.1073/pnas.1104790108

Eugeni Piller, L., Glauser, G., Kessler, F., and Besagni, C. (2014). Role of plastoglobules in metabolite repair in the tocopherol redox cycle. Front. Plant Sci. 5:298. doi: 10.3389/fpls.2014.00298

Gonzalez-Jorge, S., Ha, S. H., Magallanes-Lundback, M., Gilliland, L. U., Zhou, A., Lipka, A. E., et al. (2013). Carotenoid cleavage dioxygenase 4 is a negative regulator of beta-carotene content in Arabidopsis seeds. Plant Cell 25, 4812-4826. doi: 10.1105/tpc.113.119677

Hiltbrunner, A., Bauer, J., Vidi, P. A., Infanger, S., Weibel, P., Hohwy, M., et al. (2001). Targeting of an abundant cytosolic form of the protein import receptor at Toc159 to the outer chloroplast membrane. J. Cell Biol. 154, 309-316. doi: $10.1083 /$ jcb. 200104022 
Huang, F. C., Molnar, P., and Schwab, W. (2009). Cloning and functional characterization of carotenoid cleavage dioxygenase 4 genes. J. Exp. Bot. 60, 3011-3022. doi: 10.1093/jxb/erp137

Jin, J. B., Kim, Y. A., Kim, S. J., Lee, S. H., Kim, D. H., Cheong, G. W., et al. (2001). A new dynamin-like protein, ADL6, is involved in trafficking from the transGolgi network to the central vacuole in Arabidopsis. Plant Cell 13, 1511-1525. doi: $10.1105 /$ tpc.13.7.1511

Kessler, F., and Glauser, G. (2014). Prenylquinone profiling in whole leaves and chloroplast subfractions. Methods Mol. Biol. 1153, 213-226. doi: 10.1007/9781-4939-0606-2_15

Lätari, K., Wust, F., Hubner, M., Schaub, P., Beisel, K. G., Matsubara, S., et al. (2015). Tissue-specific apocarotenoid glycosylation contributes to carotenoid homeostasis in Arabidopsis leaves. Plant Physiol. 168, 1550-1562. doi: 10.1104/ pp.15.00243

Li, X. P., Björkman, O., Shih, C., Grossman, A. R., Rosenquist, M., Jansson, S., et al. (2000). A pigment-binding protein essential for regulation of photosynthetic light harvesting. Nature 403, 391-395. doi: 10.1038/35000131

Lippold, F., vom Dorp, K., Abraham, M., Holzl, G., Wewer, V., Yilmaz, J. L., et al. (2012). Fatty acid phytyl ester synthesis in chloroplasts of Arabidopsis. Plant Cell 24, 2001-2014. doi: 10.1105/tpc.112.095588

Lundquist, P. K., Poliakov, A., Bhuiyan, N. H., Zybailov, B., Sun, Q., and van Wijk, K. J. (2012). The functional network of the Arabidopsis plastoglobule proteome based on quantitative proteomics and genome-wide coexpression analysis. Plant Physiol. 158, 1172-1192. doi: 10.1104/pp.111. 193144

Lundquist, P. K., Poliakov, A., Giacomelli, L., Friso, G., Appel, M., McQuinn, R. P., et al. (2013). Loss of plastoglobule kinases $A B C 1 K 1$ and $A B C 1 K 3$ causes conditional degreening, modified prenyl-lipids, and recruitment of the jasmonic acid pathway. Plant Cell 25, 1818-1839. doi: 10.1105/tpc.113. 111120

Ma, J. J., Li, J., Zhao, J. B., Zhou, H., Ren, F., Wang, L., et al. (2014). Inactivation of a gene encoding carotenoid cleavage dioxygenase (CCD4) leads to carotenoidbased yellow coloration of fruit flesh and leaf midvein in peach. Plant Mol. Biol. Report 32, 246-257.

Martinis, J., Glauser, G., Valimareanu, S., and Kessler, F. (2013). A chloroplast ABC1-like kinase regulates vitamin E metabolism in Arabidopsis. Plant Physiol. 162, 652-662. doi: 10.1104/pp.113.218644

Martinis, J., Glauser, G., Valimareanu, S., Stettler, M., Zeeman, S. C., Yamamoto, H., et al. (2014). ABC1K1/PGR6 kinase: a regulatory link between photosynthetic activity and chloroplast metabolism. Plant J. 77, 269-283. doi: 10.1111/tpj. 12385

Martinis, J., Kessler, F., and Glauser, G. (2011). A novel method for prenylquinone profiling in plant tissues by ultra-high pressure liquid chromatography-mass spectrometry. Plant Methods 7:23. doi: 10.1186/1746-4811-7-23

Murashige, T., and Skoog, F. (1962). A revised medium for rapid growth and bio assays with tobacco tissue cultures. Physiol. Plant. 15, 473-497. doi: 10.1111/j. 1399-3054.1962.tb08052.x

Naested, H., Holm, A., Jenkins, T., Nielsen, H. B., Harris, C. A., Beale, M. H., et al. (2004). Arabidopsis VARIEGATED 3 encodes a chloroplast-targeted, zinc-finger protein required for chloroplast and palisade cell development. J. Cell Sci. 117, 4807-4818. doi: 10.1242/jcs.01360

Ohmiya, A., Kishimoto, S., Aida, R., Yoshioka, S., and Sumitomo, K. (2006). Carotenoid cleavage dioxygenase (CmCCD4a) contributes to white color formation in Chrysanthemum petals. Plant Physiol. 142, 1193-1201. doi: 10. 1104/pp.106.087130

Rodrigo, M. J., Alquezar, B., Alos, E., Medina, V., Carmona, L., Bruno, M., et al. (2013). A novel carotenoid cleavage activity involved in the biosynthesis of Citrus fruit-specific apocarotenoid pigments. J. Exp. Bot. 64, 4461-4478. doi: $10.1093 /$ jxb/ert260
Rottet, S., Besagni, C., and Kessler, F. (2015). The role of plastoglobules in thylakoid lipid remodeling during plant development. Biochim. Biophys. Acta Bioenergetics 1847, 889-899. doi: 10.1016/j.bbabio.2015.02.002

Rubio, A., Rambla, J. L., Santaella, M., Gomez, M. D., Orzaez, D., Granell, A., et al. (2008). Cytosolic and plastoglobule-targeted carotenoid dioxygenases from Crocus sativus are both involved in beta-ionone release. J. Biol. Chem. 283, 24816-24825. doi: 10.1074/jbc.M804000200

Ruiz-Sola, M. A., and Rodríguez-Concepción, M. (2012). Carotenoid biosynthesis in Arabidopsis: a colorful pathway. Arabidopsis Book 10:e0158. doi: 10.1199/tab. 0158

Scholl, R. L., May, S. T., and Ware, D. H. (2000). Seed and molecular resources for Arabidopsis. Plant Physiol. 124, 1477-1480. doi: 10.1104/pp.124.4.1477

Smith, M. D., Schnell, D. J., Fitzpatrick, L., and Keegstra, K. (2003). In vitro analysis of chloroplast protein import. Curr. Protoc. Cell Biol. Chapter 11:Unit11.16. doi: 10.1002/0471143030.cb1116s17

Spicher, L., and Kessler, F. (2015). Unexpected roles of plastoglobules (plastid lipid droplets) in vitamin K1 and E metabolism. Curr. Opin. Plant Biol. 25, 123-129. doi: 10.1016/j.pbi.2015.05.005

Springer, A., Acker, G., Bartsch, S., Bauerschmitt, H., Reinbothe, S., and Reinbothe, C. (2015). Differences in gene expression between natural and artificially induced leaf senescence in barley. J. Plant Physiol. 176, 180-191. doi: 10.1016/j.jplph.2015.01.004

Tevini, M., and Steinmüller, D. (1985). Composition and function of plastoglobuli: II. Lipid composition of leaves and plastoglobuli during beech leaf senescence. Planta 163, 91-96. doi: 10.1007/BF00395902

Vidi, P. A., Kanwischer, M., Baginsky, S., Austin, J. R., Csucs, G., Dörmann, P., et al. (2006). Tocopherol cyclase (VTE1) localization and vitamin E accumulation in chloroplast plastoglobule lipoprotein particles. J. Biol. Chem. 281, 11225-11234. doi: 10.1074/jbc.M511939200

Walter, M. H., Floss, D. S., and Strack, D. (2010). Apocarotenoids: hormones, mycorrhizal metabolites and aroma volatiles. Planta 232, 1-17. doi: 10.1007/ s00425-010-1156-3

Wellburn, A. R. (1994). The spectral determination of chlorophyll-a and chlorophhyll-b, as well as total carotenoids, using various solvents with spectrophotometers of different resolution. J. Plant Physiol. 144, 307-313.

Ytterberg, A. J., Peltier, J. B., and van Wijk, K. J. (2006). Protein profiling of plastoglobules in chloroplasts and chromoplasts. A surprising site for differential accumulation of metabolic enzymes. Plant Physiol. 140, 984-997. doi: $10.1104 /$ pp.105.076083

Zbierzak, A. M., Kanwischer, M., Wille, C., Vidi, P. A., Giavalisco, P., Lohmann, A., et al. (2010). Intersection of the tocopherol and plastoquinol metabolic pathways at the plastoglobule. Biochem. J. 425, 389-399. doi: 10.1042/ BJ20090704

Zhang, B., Liu, C., Wang, Y. Q., Yao, X., Wang, F., Wu, J. S., et al. (2015). Disruption of a CAROTENOID CLEAVAGE DIOXYGENASE 4 gene converts flower colour from white to yellow in Brassica species. New Phytol. 206, 1513-1526. doi: $10.1111 /$ nph.13335

Conflict of Interest Statement: The authors declare that the research was conducted in the absence of any commercial or financial relationships that could be construed as a potential conflict of interest.

Copyright (c) 2016 Rottet, Devillers, Glauser, Douet, Besagni and Kessler. This is an open-access article distributed under the terms of the Creative Commons Attribution License (CC BY). The use, distribution or reproduction in other forums is permitted, provided the original author(s) or licensor are credited and that the original publication in this journal is cited, in accordance with accepted academic practice. No use, distribution or reproduction is permitted which does not comply with these terms. 